\title{
DESIGN, SIMULATION, AND CONTROL OF A TELEOPERATED OMNIDIRECTIONAL GROUND VEHICLE
}

\author{
Andrew D. Niedert \\ Richard C. Hill* \\ Nassif E. Rayess \\ Department of Mechanical Engineering \\ University of Detroit Mercy \\ Detroit, Michigan 48221-3038
}

\begin{abstract}
This paper describes the design, construction, and simulation of a prototype, teleoperated, omnidirectional robotic ground vehicle. The design of a dynamic control system to assist the human operator of the vehicle is also presented. This work sought to test the feasibility of a novel vehicle architecture and to develop a dynamic multi-body simulation tool to assist in the development of future iterations of such a vehicle. The vehicle design seeks to achieve highspeed, omnidirectional mobility, and modest off-road capability. This paper presents results from the physical operation and simulation of the vehicle as well as describing some future work to achieve improved performance of the vehicle system.
\end{abstract}

\section{INTRODUCTION}

This paper describes the design, construction, and simulation of a prototype, teleoperated robotic ground vehicle. This vehicle was originally designed for application to the inspection of the undercarriage of military vehicles such as the Humvee. This necessitated that the vehicle be relatively low-profile, quick, maneuverable, and operable at a distance in order to keep the human driver safe. Among the robotic solutions to this application fielded to date is the OmniDirectional Inspection System (ODIS), which has three or four wheels in a very small package with a height of a few inches [1]. The speed and direction of each wheel is independently controlled by electric motors. As a result of its

\footnotetext{
*Address all correspondence to this author, hillrc@udmercy.edu.
}

low profile, ODIS is unable to traverse terrain that deviates much from a smooth paved parking lot. This deficiency motivated the design of the robot described in this work.

Speed, mobility, and the ability to negotiate uneven terrain are great assets for a robotic vehicle and provide for applicability beyond just undercarriage inspection. Other relevant applications include moving containers in a congested factory or shipping port, navigating closed buildings for the purposes of search and exploration or assisting a human in a home or hospital environment, or disaster recovery in rough, unstructured environments.

In the pursuit of the goal of maneuverability, we sought to design a vehicle that is omnidirectional, that is, a vehicle that can move in any direction without first needing to rotate the vehicle chassis. Another way to think of an omnidirectional vehicle is that it has a turning radius of zero. Such operation is not possible from Ackermann-steered vehicles (such as an automobile). There are several approaches that exist in the literature for achieving the goal of omnidirectional operation.

A well-known class of vehicles that can achieve a zeroturn radius, but not omnidirectional motion, are skidsteered vehicles. One such type are tracked vehicles, like a tank. These vehicles have two wheels (or tracks) with their axes of rotation aligned. Rotating these wheels with different velocities steers the vehicle. However, a skid-steered vehicle cannot move in any direction without first reorienting itself. Therefore, such a vehicle may be slower to maneuver than a true omnidirectional vehicle. The wheels in a skidsteered vehicle also require high levels of scrubbing that can 
be inefficient and can put significant wear and strain on the vehicle components.

Many approaches for achieving omnidirectional motion rely on specialized wheels, including the universal wheel [2] and the Mecanum wheel [3]. These wheels are similar in that they both consist of a large driven wheel with smaller passive wheels on the periphery. A disadvantage of employing these wheels is that the passive wheels on the periphery have small radii and are usually constructed of hard, non-compliant material. These facts mean that it is difficult for the wheels to maintain the generation of tractive forces while traversing even mildly uneven terrain. The smaller contact area provided by these wheels can also limit their load-bearing ability and can lead to binding, especially when the environment includes small particles and dirt. Additionally, many maneuvers that are accomplished employing these wheels require the passive motion of the individual wheels of a vehicle to "fight" against one another and hence their use can be somewhat inefficient. Another specialized wheel that exists for achieving omnidirectional motion is the ball wheel [4]. This type of wheel suffers from some of the same limitations as the universal and Mecanum wheels.

An approach that allows the use of conventional wheels is to use steerable wheels that employ one actuator to drive the wheel and a separate actuator to steer the wheel. A specific variant where the wheel is castored is called the active castor wheel [5]. Employing two such wheels can achieve true omnidirectional motion, however, significant friction and scrubbing can occur when the wheel is re-oriented about its vertical axis. This may be especially troublesome for heavy loads or for vehicles with wide tires.

An alternative that was chosen for the vehicle described in this paper is the Active Split Offset Castor (ASOC) design [6]. The ASOC design employs two independently driven wheels with aligned rotational axes offset from a vertically oriented hinge joint. The ASOC design is illustrated in Fig. 1 where the offset distance is $S$ and the split distance between the two wheels is $D$. The use of such a design is able to achieve omnidirectional motion with reduced scrubbing as compared to the steerable wheel designs mentioned previously. Furthermore, an ASOC design with the same number of actuators as a steerable wheel is able to achieve twice as much tractive force since each module has two driven wheels. The ASOC design also affords the use of conventional wheels thereby avoiding some of the limitations of the vehicle architectures based on specialized wheels. The vehicle described in this paper employs three ASOC modules as shown in Fig. 1.

Several other vehicles have been described in the literature that leverage the advantages offered by ASOC wheel modules. The application for which the ASOC design was originally designed is a robotic mobility aid for the elderly [7]. This device employs two active ASOC mod-

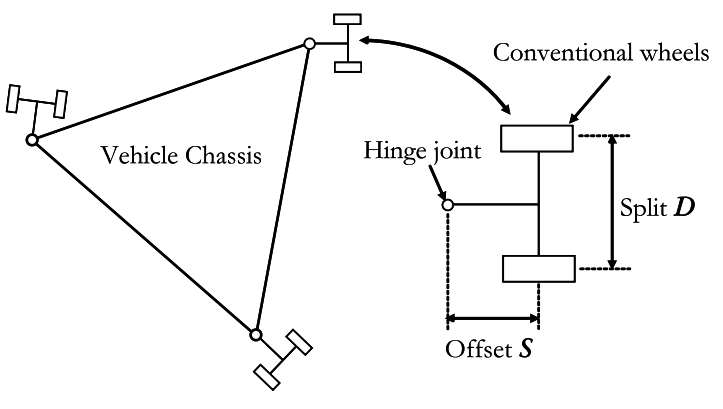

Figure 1. VEHICLE ARCHITECTURE WITH ACTIVE SPLIT OFFSET CASTOR (ASOC) MODULES.

ules along with two passive wheels and was designed to autonomously follow a given trajectory. The performance of the device was validated experimentally and the effect of different ASOC geometries was investigated analytically. A second vehicle employing ASOC modules has been constructed for the purposes of emulating proximity operations of small spacecraft [8]. The base of this vehicle employs the same basic architecture as our vehicle, three ASOC modules connected to a rigid chassis. A kinematic control law and a dynamic input-output feedback control law have been developed where the primary concern is controlling the mobile base to track a given trajectory. It is assumed that the inertial velocity of the vehicle mass center is measurable. A dynamic simulation is also developed, though it does not consider frictional forces. The design of a final vehicle employing four symmetrically spaced ASOC modules is described in [9]. The work of [9] discusses in detail the vehicle design with a focus on achieving omnidirectionality and optimal performance in rough terrain. As with the other works just described, this vehicle is controlled to autonomously track a desired body path and assumes inertial measurements are available (or can be estimated). The performance of this vehicle is validated in simulation.

The vehicle as designed in this paper differs from other works in that it is designed to be controlled directly by a human operator. Even though the vehicle is not autonomous, it was determined that a level of dynamic control was needed to assist the driver. The control scheme, however, does not need access to the inertial position or velocity of the vehicle. In addition to the design and construction of the physical vehicle, the work of this paper also developed a dynamic, multi-body simulation that includes a model of the forces at the road/tire interface. The remainder of this paper describes the design, simulation, and control of our teleoperated robotic ground vehicle. The performance of the vehicle and simulation are validated within the paper by experimental data and the paper concludes with a summary and some directions for future work. 


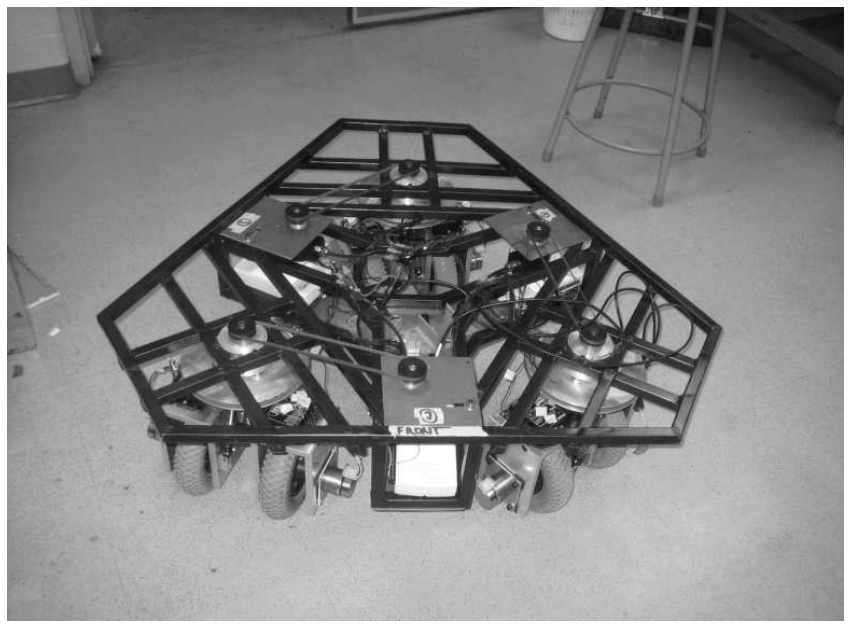

Figure 2. VEHICLE CHASSIS WITH THREE PODS.

\section{OVERVIEW OF THE VEHICLE'S DESIGN}

The vehicle chassis as currently constructed is displayed in Fig. 2. The vehicle was designed to be able to clear small obstacles $(<5 \mathrm{~cm})$ and to be able to pass under a military Humvee having a standard ride height of around $40 \mathrm{~cm} \mathrm{[10].}$ Attached to the rigid chassis are three independent ASOC modules. Complete omnidirectional motion can be achieved with only two active ASOC modules and a third passive, castored pod [6]. We chose to employ three active pods in order to provide full control authority even when a wheel loses contact with the ground and is temporarily unable to generate a tractive force. A disadvantage of this redundancy is that it may make the vehicle more difficult to control.

Each wheel is independently suspended to help it maintain contact with the ground surface, even in the presence of small surface irregularities. A detail of a single pod is shown in Fig. 3. As referenced in Fig. 1, the split distance employed for each ASOC module of this vehicle is $S=16$ $\mathrm{cm}$ and the offset distance is $D=2 \mathrm{~cm}$. The rotation of each pod is measured employing an optical encoder. Each wheel is driven by a 50-Watt brushless DC motor (Maxon EC 45 Flat) and each motor is controlled by an off-the-shelf motor controller (Maxon 1-Q-EC Ampilfier DEC 50/5 Controller). The motors themselves include three Hall-effect sensors for speed control. Signals to and from the two motors on each pod are passed through a slip ring with 16 total channels. The slip rings provide an electrical connection while allowing each pod a full 360-degree range of motion relative to the vehicle chassis.

\section{CONTROL SYSTEM DESCRIPTION}

In order to translate the commands from the human driver for the motion of the vehicle as whole into commands to the vehicle's six individual motors, it was necessary to

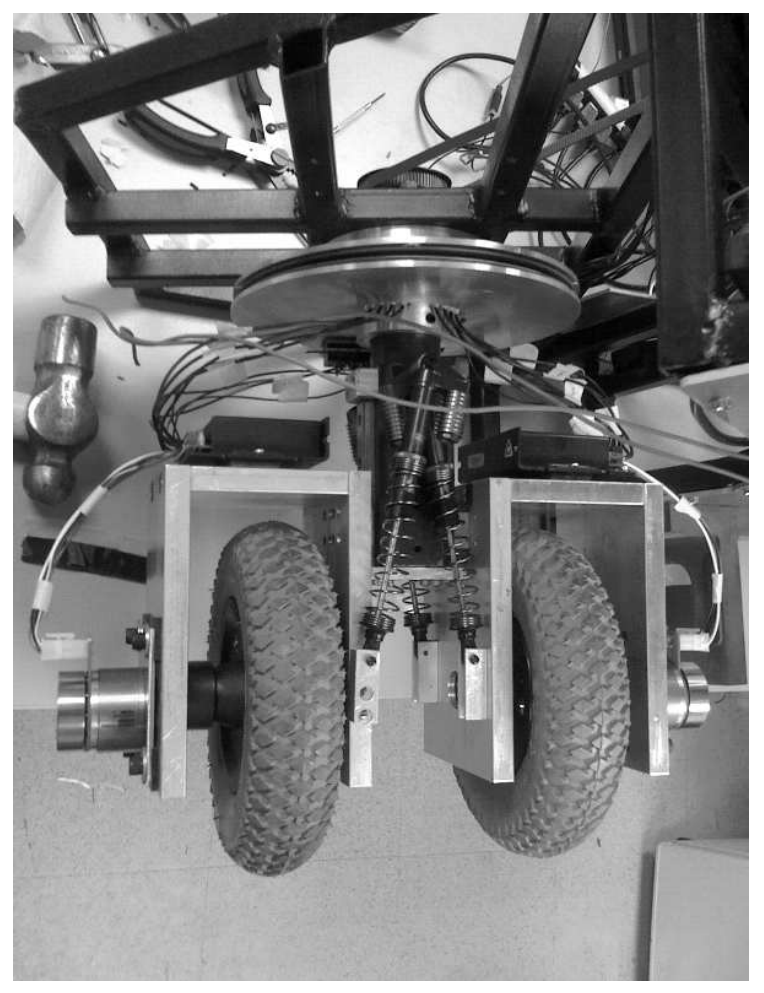

Figure 3. DETAIL OF A SINGLE POD.

develop a control system for the vehicle. In the system we have implemented, the human operator generates translational velocity and angular velocity commands via a video game console (specifically, for the Xbox 360 gaming system). These commands are transmitted via wireless communication to a laptop computer on-board the vehicle. The laptop computer runs the vehicle control software in the LabVIEW environment and employs three data acquisition (DAQ) cards for interfacing the laptop with the robot's sensors and actuators.

The initial control system developed for the vehicle was a kinematics-based control scheme. The resulting performance achieved by this approach proved to be unsatisfactory, therefore, a dynamic feedback controller was developed. In this section of the paper we describe both approaches.

\section{Kinematics-Based Control}

The desired motion of the vehicle center of mass can be directly related to the motion of the individual wheels through the use of kinematic relationships. Specifically, Eq. (1) relates the $x$ - and $y$-components of the velocity of the vehicle's center of mass $\left(V_{x}\right.$ and $\left.V_{y}\right)$ and the vehicle's angular velocity $(\omega)$ to the $x$ - and $y$-components of the velocity of the point of attachment for each of the three vehicle pods 


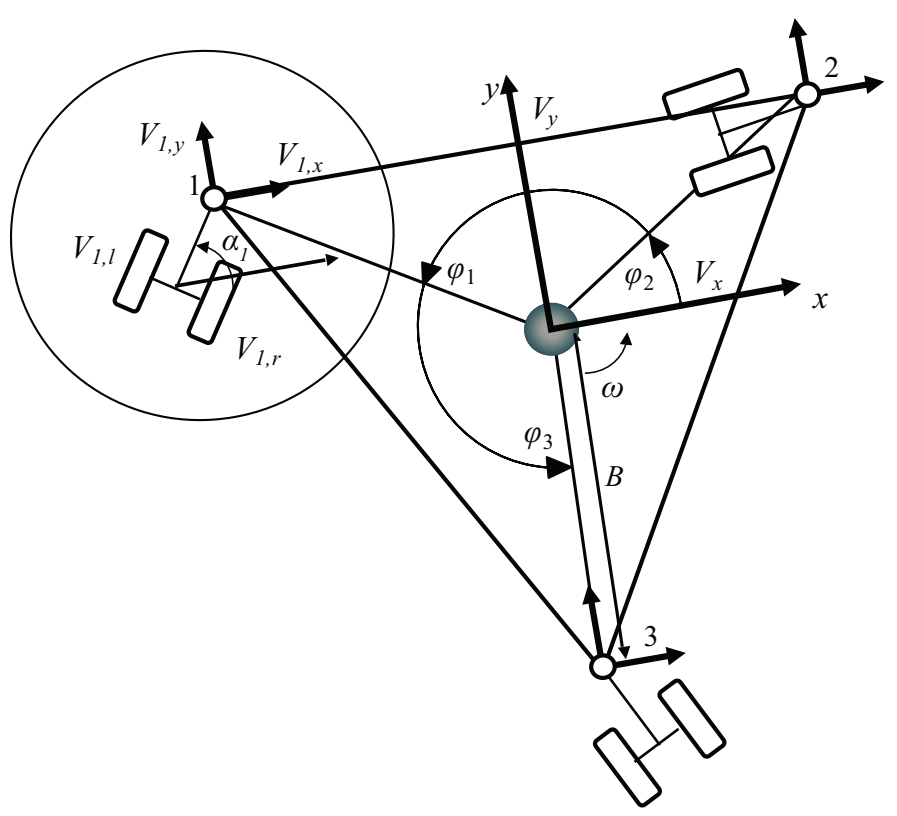

Figure 4. VEHICLE COORDINATE SYSTEMS.

$\left(V_{i, x}\right.$ and $\left.V_{i, y}\right)$ in the body frame. Figure 4 depicts the various coordinates employed throughout this section. With regard to Eq. (1), the parameter $B$ represents the distance from the vehicle center of mass to the hinge joint of each pod, while each angle $\varphi_{i}$ represents the angular location of the corresponding hinge joint relative to an axis fixed to the chassis. By locating the attachment point of each pod in this manner, the $N$ matrix is a constant and all of the velocities are expressed in a body-fixed coordinate frame. If an inertial sensor were employed to determine the heading of the vehicle, then the velocities could be expressed in an inertially-fixed frame.

$$
\left[\begin{array}{l}
V_{1, x} \\
V_{1, y} \\
V_{2, x} \\
V_{2, y} \\
V_{3, x} \\
V_{3, y}
\end{array}\right]=\left[\begin{array}{ccc}
1 & 0 & B \sin \varphi_{1} \\
0 & 1 & -B \cos \varphi_{1} \\
1 & 0 & B \sin \varphi_{2} \\
0 & 1 & -B \cos \varphi_{2} \\
1 & 0 & B \sin \varphi_{3} \\
0 & 1 & -B \cos \varphi_{3}
\end{array}\right]\left[\begin{array}{c}
V_{x} \\
V_{y} \\
\omega
\end{array}\right]=[N]\left[\begin{array}{c}
V_{x} \\
V_{y} \\
\omega
\end{array}\right]
$$

Equation (2) then relates the velocity of the right and left wheels $\left(V_{i, r}\right.$ and $\left.V_{i, l}\right)$ of each pod to the velocity of the attachment point for that pod under the assumption that the wheel velocities are aligned with their longitudinal axes (no scrubbing). The parameters $S$ and $D$ were introduced earlier in Fig. 1. Each angle $\alpha_{i}$ represents the orientation of the corresponding pod relative to the chassis. These angles are measured via optical encoders on-board the vehicle.

$$
\begin{aligned}
{\left[\begin{array}{l}
V_{i, x} \\
V_{i, y}
\end{array}\right] } & =\left[\begin{array}{ll}
\frac{1}{2} \cos \alpha_{i}-\frac{S}{D} \sin \alpha_{i} & \frac{1}{2} \cos \alpha_{i}+\frac{S}{D} \sin \alpha_{i} \\
\frac{1}{2} \sin \alpha_{i}+\frac{S}{D} \cos \alpha_{i} & \frac{1}{2} \sin \alpha_{i}-\frac{S}{D} \cos \alpha_{i}
\end{array}\right]\left[\begin{array}{c}
V_{i, r} \\
V_{i, l}
\end{array}\right] \\
& =\left[J_{i}\right]\left[\begin{array}{c}
V_{i, r} \\
V_{i, l}
\end{array}\right]
\end{aligned}
$$

Finally, employing Eq. (1) and Eq. (2) together leads to the inverse kinematic relationship given in Eq. (3). This relationship forms the basis for the kinematics-based control law we initially implemented on the vehicle. Specifically, Eq. (3) takes as inputs the commands from the human operator $\left(\left[V_{x}, V_{y}, \omega\right]\right)$ and outputs the corresponding desired wheel velocities $\left(\left[V_{1, r}, V_{1, l}, V_{2, r}, V_{2, l}, V_{3, r}, V_{3, l}\right]\right)$. These wheel velocities then determine the angular velocity setpoints to the off-the-shelf motor controllers. These motor controllers apply an unknown speed control feedback law employing the Hall-effect sensors that came packaged with the motors.

$$
\left[\begin{array}{l}
V_{1, r} \\
V_{1, l} \\
V_{2, r} \\
V_{2, l} \\
V_{3, r} \\
V_{3, l}
\end{array}\right]=\left[\begin{array}{ccc}
{\left[J_{1}\right]} & 0 & 0 \\
0 & {\left[J_{2}\right]} & 0 \\
0 & 0 & {\left[J_{3}\right]}
\end{array}\right]^{-1}[N]\left[\begin{array}{c}
V_{x} \\
V_{y} \\
\omega
\end{array}\right]
$$

Note that the vehicle velocity commands are given in a coordinate frame attached to the vehicle, thereby avoiding the necessity of an expensive inertial sensor. This strategy can be employed due to the fact that a human driver is, in essence, part of the feedback loop.

Under idealized conditions, this approach to control will provide the commanded motion of the vehicle. Specific assumptions that are necessary include that the vehicle geometry is known perfectly, that each wheel contact patch has minimal area, that the vehicle wheels roll without slip (or scrubbing), and that the commanded wheel speed profiles can be followed exactly. In practice we have determined that these assumptions are not reasonable. Any errors in the kinematic model are exacerbated by the fact that we are employing three ASOC modules, in other words, our system is overdetermined. We, therefore, have developed the dynamic feedback control scheme described in the following section.

\section{Dynamic Control}

The control system that we have settled on includes the kinematics-based controller described previously plus a feedback controller that closes the loop on pod angle. The kinematics-based controller adds a feedforward element to 
the controller that reacts very quickly and predominately controls the overall speed of the vehicle. The feedback portion of the controller serves to correct for disturbances and errors in the kinematic model. The feedback controller, in particular, helps to achieve the vehicle heading desired by the human driver. The human driver can effectively control the speed of the vehicle, but due to the wheel scrubbing and bumps, etc., the driver cannot achieve the desired heading without assistance. We chose to close the loop on pod angle since it is a quantity that is readily available (measured by encoders) and because the dynamics of the pods are relatively quick. Though the significant stiction present in the slip rings has proved challenging to address. Figure 5 illustrates the structure of the dynamic control scheme employed on-board the vehicle.

Generation of the setpoint for each pod controller is calculated based on Eq. 4 where it is assumed that the wheels of each pod align with the desired direction of motion. This situation corresponds to negligible scrubbing of the wheels.

$$
\alpha_{i, \text { desired }}=\arctan \left(\frac{V_{i, y}}{V_{i, x}}\right)
$$

In order to decouple the feedforward and feedback portions of the controller, the $N$ matrix employed in the feedforward controller (Eq. 1) employs the desired pod angles rather than the actual pod angles $\left(\alpha_{i}\right)$. This choice helps to make the process of tuning the controller more straightforward.

The structure of the feedback portion of the pod controller was ultimately chosen to be a proportional-integralderivative (PID) controller because it was straightforward to implement and intuitive to tune. Since the dynamic model of the vehicle is uncertain and nonlinear (trigonometric functions, generation of road forces, friction, etc.), the controller was tuned empirically to achieve satisfactory performance. An integrator anti-windup scheme was implemented to help address the significant stiction contributed by the slip rings.

\section{DYNAMIC MULTI-BODY SIMULATION}

In order to aid the development of the current and future iterations of the vehicle, we also sought to develop a dynamic multi-body simulation to qualitatively predict vehicle performance. Such a simulation provides a safe environment in which different vehicle control strategies can be quickly developed. The simulation environment also provides a means for testing alternative vehicle geometries and configurations without the need to physically alter the vehicle.

In order to speed the simulation's development, it was chosen to implement the simulation using the physical mod- eling tools of the Simscape addition to Simulink. This software package allows the creation of a simulation model by defining its physical characteristics, rather than from a mathematical model. This is especially helpful in this case since the multi-body nature of the vehicle makes the generation of the mathematical model challenging. In the Simscape environment we, for example, were able to define the geometric and inertia properties of the chassis and each of the pods, as well as the properties of the hinge joints that connect the various bodies. There was no need for us to explicitly derive the equations of motion for any of the bodies, this in essence was done behind the scenes by the Simscape software. In addition to simulating the vehicle dynamics, the Simscape software also generates an animation of the vehicle's motion. An animation for one specific scenario is depicted in Fig. 11.

The simulation we developed is shown at a high level in Fig. 6 . The simulation only models the motion of the vehicle in a two-dimensional plane (no roll or pitch). After creating the physical vehicle within Simscape, models for the various friction and drag forces (torques) were implemented based on some simple experiments performed on the vehicle. The simulation models the generation of tire forces employing a Pacejka-type model [11], where the various parameters of the Magic Formula were also estimated based on simple experiments. The dynamics of the closed-loop wheel/motor subsystems were generated empirically since the structure of the motor controllers is unknown. In general, these experiments were far from perfect, but did seem to generate models that are qualitatively correct.

\section{Tire Model}

As stated above, the Magic Formula [11] was employed for modeling the forces (lateral and longitudinal) generated at the road/tire interface. The general form of the force model is displayed in Eq. 5 where the coefficients $(B, C$, $D$, and $E$ ) were chosen to fit experimental data. In the case of the lateral tire force (parallel to the wheel's axle), the magnitude of the force is modeled as a function of the side-slip angle where side-slip angle is the angle between the tire's actual direction of motion and the direction that the tire is pointing.

$$
F(x)=D \sin (C \arctan (B x-E(B x-\arctan (B x))))
$$

Figure 7 displays the lateral force data used to set the coefficients employed in the accompanying tire model. The data was generated by conducting a static experiment on the vehicle where a force transducer was attached to the chassis and pulled at a constant angle in relation to the chassis at a steady speed. The encoders attached to the pods were utilized to identify the specific angles at which 


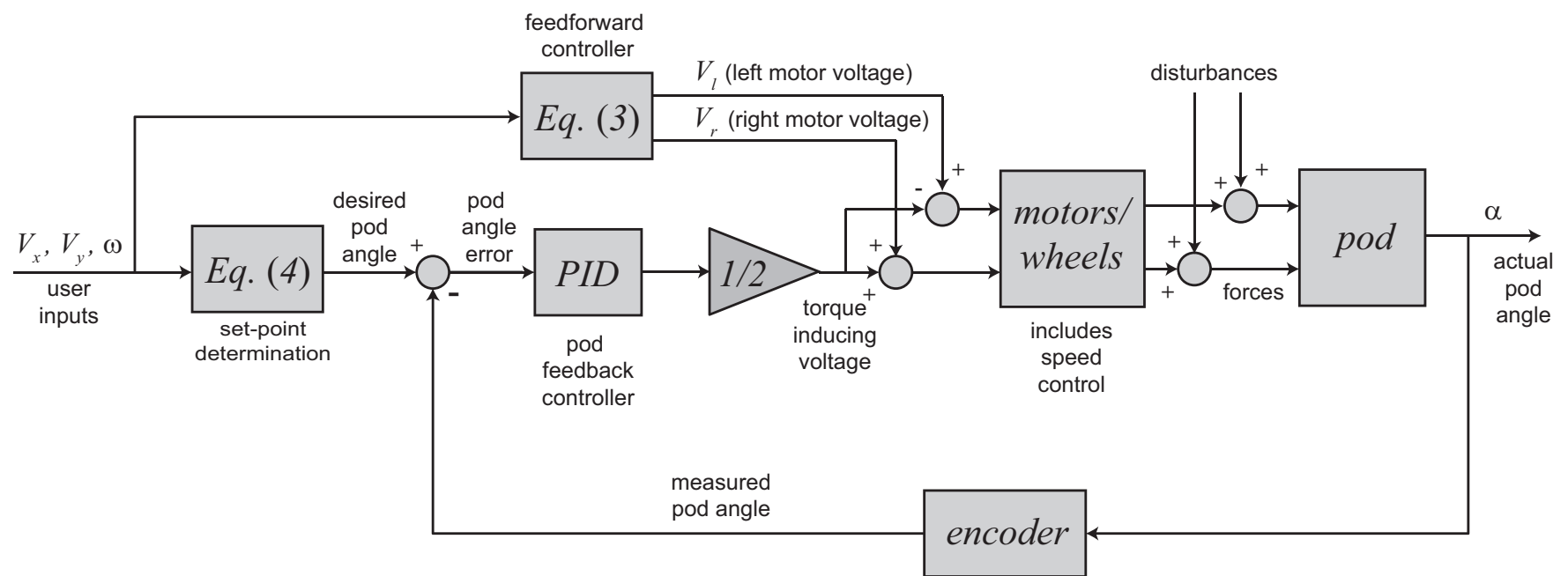

Figure 5. DYNAMIC POD CONTROL SYSTEM FOR A SINGLE POD.

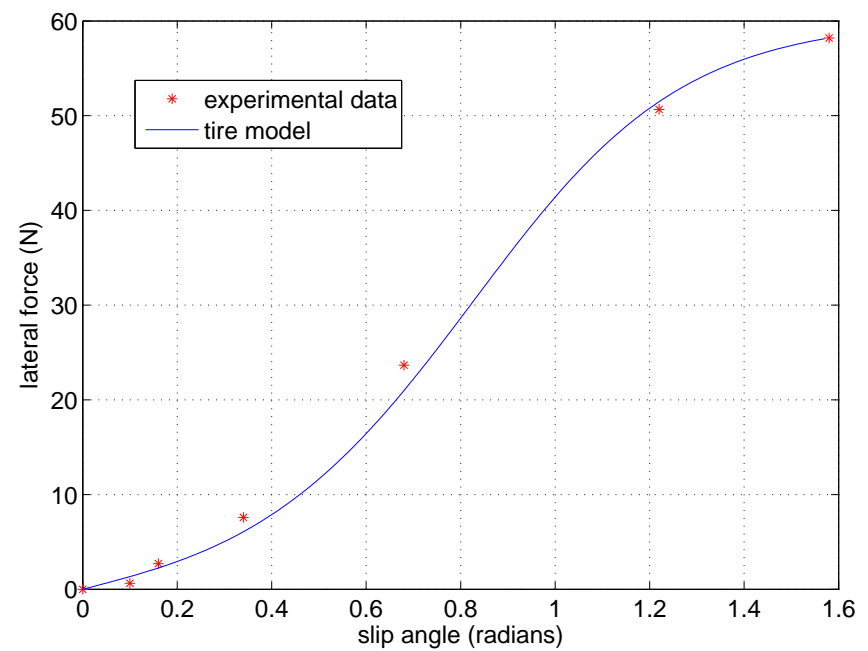

Figure 7. LATERAL TIRE MODEL WITH EXPERIMENTAL DATA.

the pod was pulled. These angles represent the various slip angles over which the vehicle may operate. The measured pulling force exactly balances the tire force when dragged at constant speed. The experiment was performed on the same ground surface as subsequent vehicle-level tests. The model shown in Fig. 7 is generated by Eq. 5 , where $B=0.2$, $C=1.08, D=60$, and $E=-250$.

The longitudinal tire forces were also modeled by Eq. 5 , except here the tractive force is modeled as a function of the longitudinal slip ratio. Longitudinal slip is a measure of the difference between the tire's actual velocity $(v)$ and the velocity it would have if it were rolling without slip $(r \omega)$. Longitudinal slip in general will be positive under acceleration and negative under braking and is often expressed as a percentage or a ratio. For example, we were primarily concerned with the longitudinal forces generated under acceleration since we do not actively brake the wheels of our vehicle, therefore, we expressed the slip ratio as shown in Eq. 6.

$$
\text { slip ratio }=\frac{r \omega-v}{r \omega}
$$

The experimental data employed for determining the coefficients of the longitudinal tire model are shown in Fig. 8. This data was recorded over several experiments where the pods were locked and the vehicle was driven with two of the three pods active. By disabling the motors driving the wheels in one of the pods, we were able to estimate the vehicle speed from the non-driven wheels employing the assumption that those wheels were rolling with minimal slip. The total longitudinal force of the four driving wheels was estimated from the vehicle's acceleration, again as determined from the non-driven wheel speeds (less the estimated drag and rolling resistance forces). This means that force estimates were in essence averaged over the four driven wheels. The experiments were performed for different levels of acceleration to generate data over a range of slip ratios. These experiments were conducted cheaply 


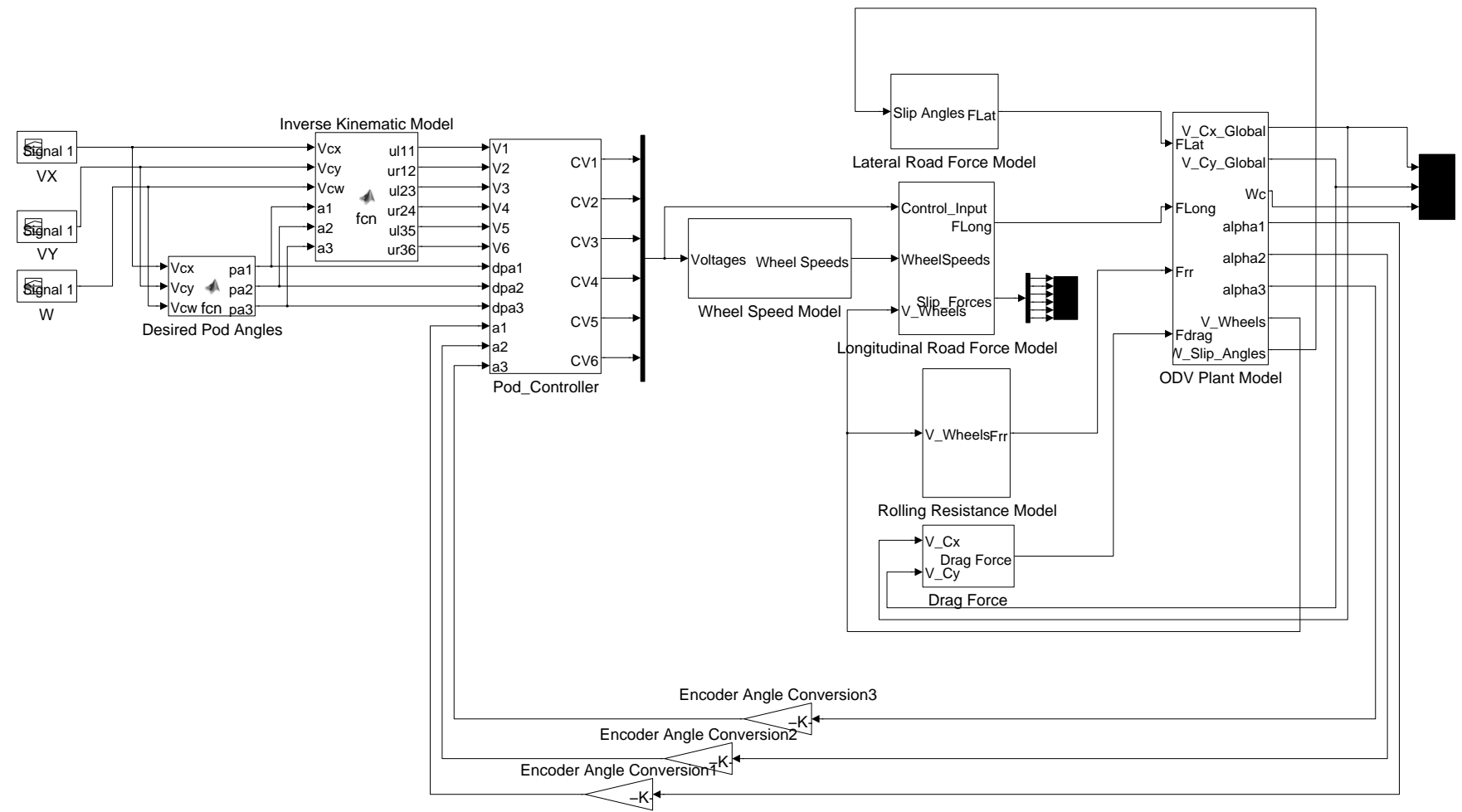

Figure 6. MULTI-BODY DYNAMIC SIMULATION IMPLEMENTED IN SIMSCAPE.

and quickly, and while subject to quite a bit of error as evidenced by the scatter in the data, did produce repeatable results showing the approximately linear relationship between tractive force and slip ratio at small levels of slip. Dynamic experiments at high levels of slip were difficult to perform, however, we were able to get a sense of performance at high levels of slip by measuring the force generated by the wheels when they are locked.

The resulting longitudinal tire model is represented by Eq. 5 with $B=3.5, C=0.8, D=70$, and $E=-0.2$. The results of this model are depicted in Fig. 8 alongside the data used to arrive at the aforementioned coefficients. The longitudinal model over the full range of slip ratios is shown in Fig. 9.

\section{EXPERIMENT AND SIMULATION DATA}

Several experiments were run on the physical vehicle to demonstrate the vehicle's performance and the validity of the multi-body dynamic simulation that had been developed. One set of tests consisted of accelerating and decelerating the vehicle in a straight line (pods locked in alignment). These tests demonstrated the accuracy of the longitudinal portion of the simulation model. The results from one such test are shown in Fig. 10. Velocity data for the actual vehicle was estimated from wheel-speed measurements for two non-driven wheels as was done in generating the lon-

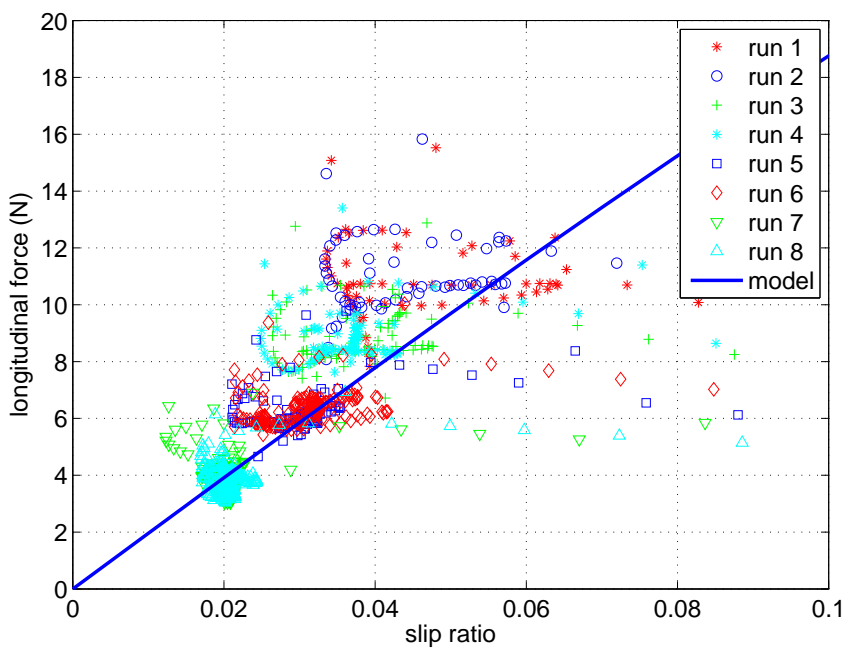

Figure 8. EXPERIMENTAL DATA EMPLOYED FOR DERIVING THE LONGITUDINAL TIRE MODEL.

gitudinal tire model. The physical control input employed for this experiment (command on $V_{y}$ ) was then employed as the input to the simulation. The minor differences exhibited between the experimental data and the simulation data are likely attributable primarily to errors in the tire model and errors in the closed-loop motor system model.

A second experiment performed to evaluate the per- 


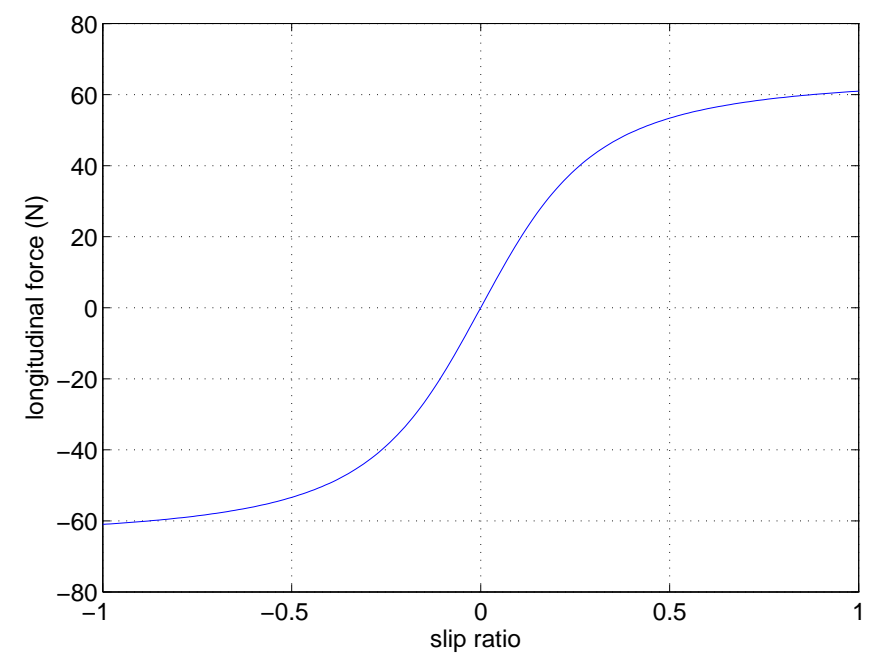

Figure 9. LONGITUDINAL TIRE MODEL.

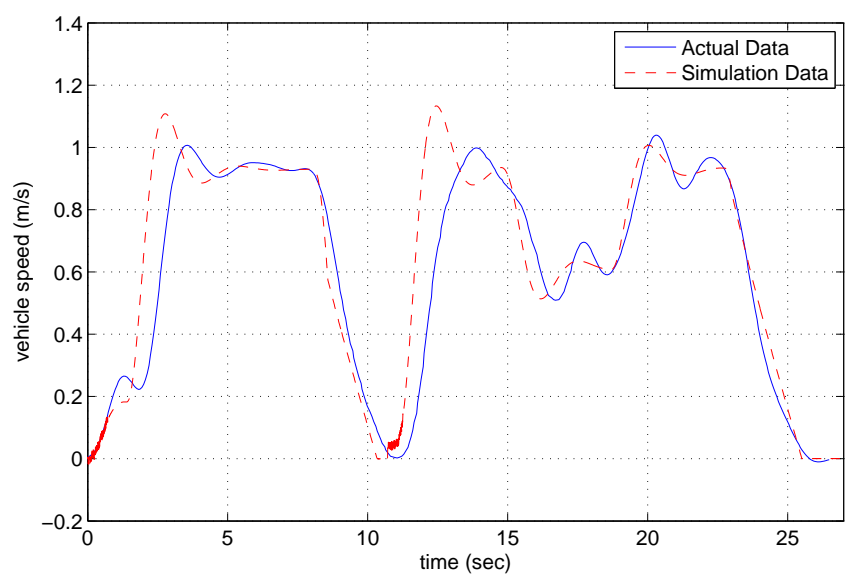

Figure 10. COMPARISON OF EXPERIMENTAL AND SIMULATION VEHICLE SPEED FOR LONGITUDINAL ACCELERATION AND DECELERATION.

formance of the control system and of the simulation as whole (including lateral dynamics) involved driving the vehicle through the S-course shown in Fig. 11. Driving the actual vehicle through this course was possible, though quite challenging, due to the significant stiction present in the slip rings. In order to give the control system time to overcome the stiction in the slip rings, the human driver had to begin commanding a turn multiple seconds before the turn was desired. Furthermore, the stiction made it difficult to make fine corrections to the vehicle's heading. During an experiment in which the slip ring on the trailing pod was removed and the various wires connected directly to the motor controllers, the human driver was able to traverse the course quite easily without having to anticipate an upcoming turn.

Data of the actual vehicle heading for a successful

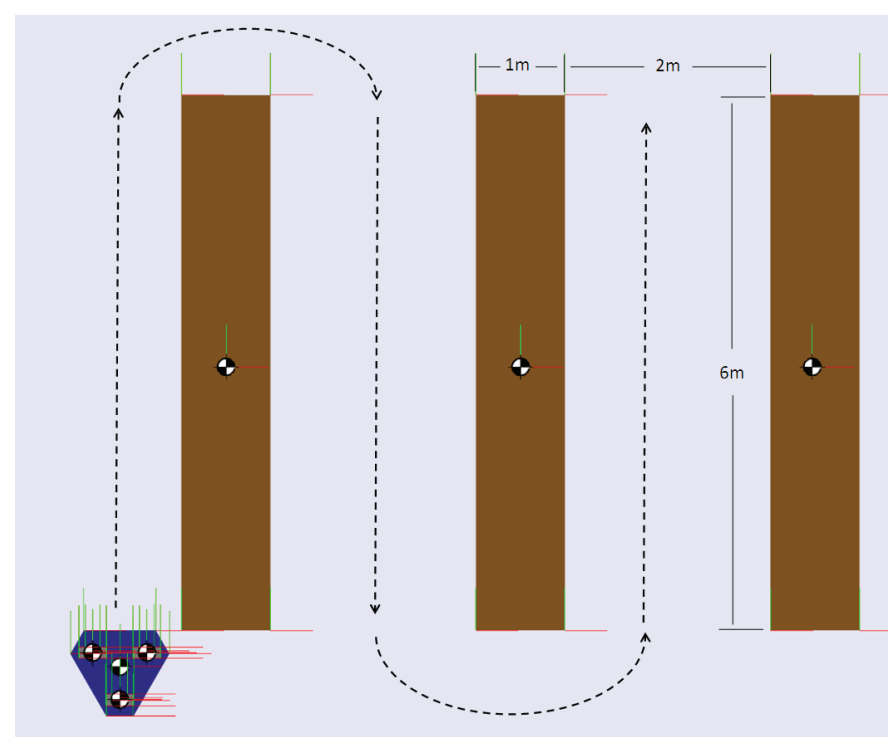

Figure 11. S-COURSE EMPLOYED IN SECOND EXPERIMENT.

traversal of the aforementioned S-course is shown in Fig. 12. This run was performed with all three slip rings connected and the heading data was recorded via an inertial measurement unit (IMU). The control signals for the depicted run were recorded and employed as inputs to the multi-body vehicle simulation. The results of this simulation are included within Fig. 12. This figure demonstrates that while the experimental and simulation data don't match particularly well, there is a qualitative agreement that is apparent. The actual vehicle data corresponds to a run where the vehicle traversed the shown course at a constant speed of approximately $1 \mathrm{~m} / \mathrm{s}$. The vehicle was run at about $1 / 5$ of its maximum possible speed in order to avoid damage to the motors which are undersized for the vehicle in its current configuration. The vehicle in simulation was not able to traverse the course for the exact control inputs applied to the actual vehicle. This is due in part to errors in the model, imperfect ground, weight shift of the vehicle, etc. If a driver model was included to close the loop on the inertial position of the vehicle, the vehicle would also be able to traverse the course in simulation.

Even though the simulation results are somewhat inaccurate, the results presented above demonstrate a qualitative level of performance that indicates that the simulation could be quite useful, especially with the inclusion of a driver model. In particular, this simulation could be employed for testing different control strategies and vehicle geometries. The simulation is especially useful for estimating quantities that are not easily measured on the actual vehicle, for example, the forces generated at the road/tire interface. 


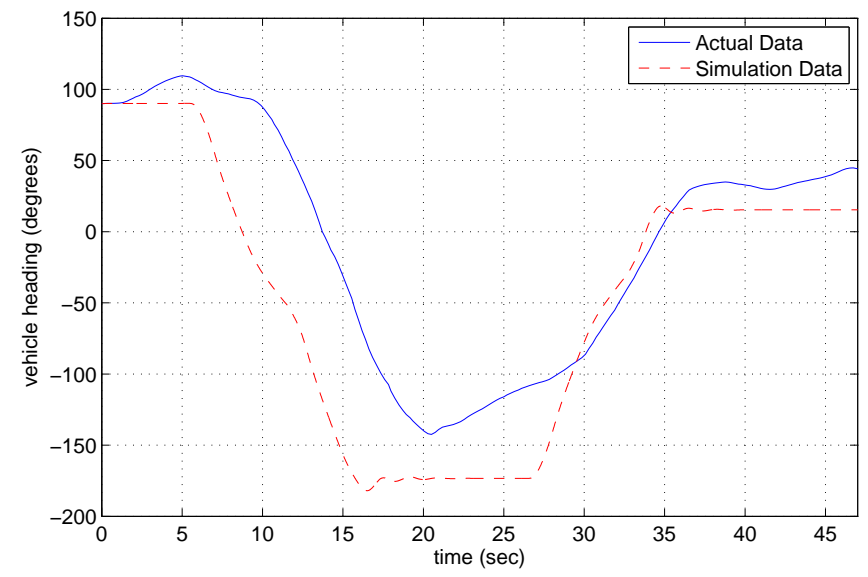

Figure 12. COMPARISON OF EXPERIMENTAL AND SIMULATION VEHICLE HEADING DURING TRAVERSAL OF S-COURSE.

\section{CONCLUSION AND FUTURE WORK}

In this paper, the design and construction of a teleoperated, omnidirectional, robotic ground vehicle was described. The development of a dynamic control algorithm to assist the driver in maintaining desired vehicle heading was explained and actual vehicle data was shown demonstrating the operability of the vehicle. Additionally, the development of a multi-body dynamic simulation was described and shown to be able to predict vehicle behavior that agrees qualitatively with actual data from the physical vehicle.

One of the primary, original aims of this vehicle design was to achieve modest off-road capability. To this point, challenges with under-sized motors and slip-ring friction have limited the amount of experimental testing that we have been able to undertake. As such, we have not been able to test the vehicle's performance in more rugged environments. A future task will be to address these limitations so that the vehicle may be tested under such conditions.

Specifically, it is hoped that another iteration of the vehicle can be built that addresses the slip-ring stiction and under-powered motors. Specific modifications to the design would be to employ wireless communication for the nonpower signals being passed to and from the motor and motor controllers for each pod. Slip rings would still be needed to transmit power, but the drastic reduction in the number of channels should greatly reduce the friction generated by the slip rings. This change in slip ring implementation will also help with the motors being underpowered as it has been observed that the motors don't overheat as easily when they don't have to overcome the significant friction of the slip rings. The motor problems could also be addressed by employing different motors, or by reducing the overall weight of the vehicle. Additionally, it is planned to develop a more sophisticated control strategy based on a dynamic model of the vehicle. This new control algorithm will replace the empirically tuned PID-based pod controllers, and may employ an inertial sensor.

\section{ACKNOWLEDGMENT}

This work was in part supported by the the U.S. Army Tank Automotive Research, Development and Engineering Center (TARDEC) and the University of Detroit Mercy's Faculty Grant Incentive Program (FGIP). Gratitude is expressed to students Alex Szmatula, Li Guan, and Yazan Aljeroudi for their contributions to the overall project.

\section{REFERENCES}

[1] Flann, N. S., Moore, K. L., and Lili, M., 2002. "A small mobile robot for security and inspection operations". Control Engineering Practice, 10(11).

[2] Ferriere, L., and Raucent, B., 1998. "ROLLMOBS, a new universal wheel concept". In 1998 IEEE International Conference on Robotics and Automation.

[3] Muir, P., and Neuman, C., 1987. "Kinematic modeling for feedback control of an omnidirectional wheeled robot". In 1987 IEEE International Conference on Robotics and Automation.

[4] West, M., and Asada, H., 1997. "Design of ball wheel mechanisms for omnidirectional vehicles with full mobility and invariant kinematics". Journal of Mechanical Design, $\mathbf{1 1 9}(2)$.

[5] Wada, M., and Mori, S., 1996. "Holonomic and omnidirectional vehicle with conventional tires". In IEEE International Conference on Robotics and Automation.

[6] Yu, H., Dubowsky, S., and Skwersky, A., 2004. "Omnidirectional mobility using active split offset castors". Journal of Mechanical Design, 126(5).

[7] Spenko, M., Yu, H., and Dubowsky, S., 2002. "Analysis and design of an omnidrectional platform for operation on non-ideal floors". In 2002 IEEE International Conference on Robotics and Automation.

[8] Bai, X., Davis, J., Doebbler, J., Turner, J., and Junkins, J., 2008. "Modeling, control and simulation of a novel mobile robotic system". Engineering Letters, 16(2).

[9] Udengaard, M., and Iagnemma, K., 2009. "Analysis, design, and control of an omnidirectional mobile robot in rough terrain". Journal of Mechanical Design, 131(12).

[10] On the WWW, 2011. AM General LLC - Mobility Solutions for the 21st Century, August. URL www . amgeneral. com/vehicles/hmmwv/features . php.

[11] Pacejka, H., and Bakker, E., 1992. "The magic tyre formula model". Vehicle System Dynamics: International Journal of Vehicle Mechanics and Mobility, 21(1). 